\title{
Metagenomic next-generation sequencing of radial ultrasound bronchoscopy-guided "cocktail" specimens as an efficient method for the diagnosis of focal pulmonary infections: a randomised study
}

\author{
Ying Chen, Xiaopei Yan, Ning Li, Qin Zhang, Yantian Lv, Ting Ruan \\ Department of Respiratory and Critical Care Medicine, The Affiliated Suzhou Hospital of Nanjing Medical University, Suzhou Municipal Hospital, \\ Suzhou, China \\ Contributions: (I) Conception and design: T Ruan; (II) Administrative support: None; (III) Provision of study materials or patients: All authors; (IV) \\ Collection and assembly of data: Y Chen, X Yan; (V) Data analysis and interpretation: T Ruan, Y Lv, Y Chen, X Yan; (VI) Manuscript writing: All \\ authors; (VII) Final approval of manuscript: All authors. \\ Correspondence to: Ting Ruan; Yantian Lv. Department of Respiratory and Critical Care Medicine, The Headquarter of Suzhou Municipal Hospital \\ (Suzhou Hospital Affiliated to Nanjing Medical University), No. 26 Daoqian Street, Suzhou 215005, China. \\ Email: yutingkeke@163.com; lvyantian@126.com.
}

Background: To investigate the value of metagenomic next-generation sequencing (mNGS) in the diagnosis of focal pulmonary infections by using radial ultrasound bronchoscopic "cocktail" specimens.

Methods: From March 2019 to May 2020, 90 patients with focal pulmonary infections [locatable by a radial probe endobronchial ultrasound (RP-EBUS)] treated by the Department of Respiratory and Critical Care Medicine at the Affiliated Suzhou Hospital of Nanjing Medical University, Suzhou Municipal Hospital (Suzhou, China) were randomly allocated to the "cocktail" group, transbronchial brushing (TBBr) group or bronchoalveolar lavage fluid (BALF) group; 30 patients were assigned to each group. Using the extracted material, the diagnostic efficacy of the mNGS test for pathogen detection was compared across the three groups, and the effect of an antibiotic treatment on detection rate was assessed.

Results: The sensitivity of mNGS analysis in cocktail group, TBBr group and BALF group was $90 \%$ (27/30), 66.7 (20/30) and 50\% (15/30), respectively. The analysis of the sensitivity of the positive test, the detection rate of multiple pathogens, and the effect of antibiotics on the detection rate by mMS indicated that the "cocktail" group was significantly higher number of samples positive for pathogens than the TBBr group $(\mathrm{P}<0.05)$, and the TBBr group was significantly higher number of samples positive for pathogens than the BALF group $(\mathrm{P}<0.05)$.

Conclusions: "Cocktail" specimens had high sensitivity in the identification of focal pathogens, and the use of antibiotics had little effect on the results of the mNGS analysis. These clinically valuable results can be used in the diagnosis and treatment of patients with focal pulmonary infections.

Keywords: Metagenomic next-generation sequencing (mNGS); “cocktail” specimen; transbronchial brushing (TBBr); bronchoalveolar lavage fluid (BALF); focal infection; pathogens

Submitted Nov 24, 2020. Accepted for publication Feb 04, 2021.

doi: 10.21037/apm-20-2578

View this article at: http://dx.doi.org/10.21037/apm-20-2578 


\section{Introduction}

The radial probe endobronchial ultrasound (RP-EBUS) is a new type of assisted detection method that enables biopsies to be taken under the non-real-time guidance of ultrasound instrumentation. The RP-EBUS is able to clearly demonstrate local lesions on the pathway, and accurately obtain lesion specimens. It is convenient, fast, and safe (1). Clinical studies have found that as many as 100 pathogens are associated with lung infections. However, as focal infections caused by a ribonucleic acid (RNA) virus were rarely, the present study only selected deoxyribonucleic acid (DNA) for metagenomic sequencing to analyze the pathogen.

The early and rapid identification of pathogens and the rational selection of anti-infective drugs is key to improving clinical outcomes (2). Traditional microbial pathogen detection techniques, such as polymerase chain reactions (PCRs) and microbial cultures, have been widely used to diagnose pulmonary infections. However, as the detection rate of these methods is low and time-consuming, and it is difficult to achieve a highly efficient diagnosis using these methods, these methods can no longer meet clinical needs (3). Based on the continuous development of sequencing technology and bioinformatics, mature metagenomic next-generation sequencing (mNGS) has been developed that requires only a small number of samples from lesion tissues for a sequencing analysis. The identification of pathogens can be achieved by comparing the sequencing results of $\mathrm{mNGS}$ to an accurate reference genomic database (4). This emerging approach is changing how physicians diagnose and treat infectious disease, with applications spanning a wide range of areas, including antimicrobial resistance, the microbiome, human host gene expression (transcriptomics) and oncology (5). Pulmonary infection is a leading cause of death and morbidity worldwide (6). However, its diagnosis is challenging due to the multitude of possible pathogens. Hundreds of pathogens have been associated with pulmonary infections, including bacterial, viral, or fungal pathogens (7). Thus, mNGS can be a key driver for precision diagnosis of infectious diseases, advancing precision medicine efforts to personalize patient care in this field (8). As mNGS has a number of advantages, including that it has a high pathogen detection rate, is time saving, and can provide accurate information for clinical diagnosis, it has been gradually applied to the diagnosis of infectious diseases (9).

The main intrapulmonary interventional sampling methods for mNGS analysis include transbronchial lung biopsy (TBLB), transbronchial brushing (TBBr), and bronchoalveolar lavage (BAL) (10). The method for extracting genetic material from pathological specimens obtained by different extraction methods and combining them for mNGS detection is referred to as the "cocktail" method. This method helps to reduce detection costs, increases the detection rate and has been applied to clinical diagnoses (11). The sample obtained by TBLB is a tissue pellet. If the sample obtained is a representative lesion (i.e., a deep tissue infection), the sensitivity and specificity of the analysis is optimal, and it is easy to determine whether the lesion is infected or colonized. TBLB is especially advantageous for the diagnoses of Yersinia pneumoniae infections and cytomegalovirus infections. TBLB can assist in diagnosis and has the advantages of rapid onsite evaluation (ROSE) and histopathology. However, the sampling range of TBLB is limited to one or several points in the lung; thus, if representative foci are not obtained, false negatives may occur. It should be noted that the false negative rate of TBLB is higher than that of TBBr and BALF. Additionally, TBLB tissue biopsies are prone to cause complications, and have a complication rate of approximately $14 \%(12,13)$. Performing multiple TBLBs on patients who are continuously mechanically ventilated is also likely to give rise to complications, such as pneumothorax, intra-bronchial hemorrhage, and persistent oxygen desaturation. Conversely, the complication rates of $\operatorname{TBBr}(8 \%)$ (14) and BALF (6.8\%) (15) are lower than that of TBLB. As the pathological range of specimens obtained by focal infection TBLB is small, and the psychological acceptance of bronchoscopic complications is low in patients, TBLB was abandoned as a means of sampling in this study. In this study, the cocktail specimen referred to the mixture of TBBr brush head and BALF collected from target site. According to the results of a "cocktail sampling" combined with a mNGS analysis, only a very small number of patients need TBLB once again if necessary. In the present study, we performed RP-EBUS on patients with focal pulmonary infections admitted to the Respiratory Department at our Hospital from March 2019 to May 2020, and we used "cocktail" samples, TBBr samples and BALF samples as the delivering samples for DNA sequencing analysis by mNGS. We also analyzed the positive diagnosis rates of different sampling methods to comprehensively evaluate their diagnostic value in focal pulmonary infections. Metagenome second-generation sequencing (mNGS) using radial bronchoscopy cocktail specimens has a high pathogen 
Table 1 Clinical characteristic statistics of the 3 groups of patients with focal pneumonia (n)

\begin{tabular}{lccccccc}
\hline Groups & Fever & Cough & $\begin{array}{c}\text { WBC count } \\
\text { abnormal }\end{array}$ & $\begin{array}{c}\text { Neutrophil percentage } \\
\text { abnormal }\end{array}$ & $\begin{array}{c}\text { Calcineurin } \\
\text { abnormal }\end{array}$ & $\begin{array}{c}\text { CRP } \\
\text { abnormal }\end{array}$ & $\begin{array}{c}\text { Antibiotic use before } \\
\text { mNGS }\end{array}$ \\
\hline "Cocktail" group & 8 & 17 & 16 & 14 & 3 & 21 & 26 \\
TBBr group & 10 & 18 & 15 & 16 & 2 & 22 & 25 \\
BALF group & 9 & 20 & 17 & 15 & 2 & 21 & 26 \\
\hline
\end{tabular}

$\mathrm{TBBr}$, transbronchial brushing; BALF, bronchoalveolar lavage fluid; WBC, white blood cell; CRP, C-reactive protein.

detection rate, is time saving, and can provide accurate information for clinical diagnosis, and the use of antibiotics had little effect on the results of the mNGS analysis. These clinically valuable results can be used in the diagnosis and treatment of patients with focal pulmonary infections.

We present the following article in accordance with the CONSORT reporting checklist (available at http://dx.doi. org/10.21037/apm-20-2578).

\section{Methods}

\section{General Information}

From March 2019 to May 2020, 90 patients with focal pulmonary infections locatable by RP-EBUS were admitted to the Respiratory and Critical Care Medicine Department at our Hospital. These patients were allocated to the "cocktail" group, TBBr group, or BALF group using a random number table; 30 patients were assigned to each group. The "cocktail" group comprised 19 males and 11 females, aged 22 to 73 years, with an average age of $46.7 \pm 3.4$ years; the $\mathrm{TBBr}$ group comprised 20 males and 10 females, aged 24 to 75 years, with an average age of $45.8 \pm 4.6$ years; and the BALF group comprised 22 males and 8 females, aged 23 to 74 years, with an average age of $46.9 \pm 5.1$ years. The clinical data is set out in Table 1. Clinical characteristic data between the three groups were analyzed by chi-square test, which show no significant difference $(\mathrm{P}>0.05)$.

\section{Inclusion and exclusion criteria}

To be eligible to participate in the study, participants had to meet the following inclusion criteria: (I) be aged from 18 to 80 ; (II) display suspicious lesions in clinical tests; and (III) have no contraindications to bronchoscopy. The study was conducted in accordance with the Declaration of Helsinki (as revised in 2013). The study was approved by Ethical Committee of Suzhou Municipal Hospital (No.:
KL901157). All the patients and their families were informed of and agreed to participate in the study and signed the informed consent form.

Participants were not eligible to participate in the study, if they met any of the following exclusion criteria: (I) had a non-infectious disease(s); (II) had respiratory tract bleeding or a tendency to bleed; (III) had severe cardiopulmonary dysfunction; (IV) had a clear pathological diagnosis or had received an effective empirical treatment; (V) based on clinical judgment, did not require a bronchoscopy to further diagnose the etiology of the disease; and/or (VI) demonstrated poor compliance or an inability to cooperate with treatment.

\section{Sampling method}

The pre-operative evaluation of patients in the three groups included routine blood work, an electrocardiogram, and an assessment of basic vital signs. Patients' tolerability was analyzed. Based on the computerized tomography (CT) images of the chests of the patients, the course of the bronchus, the location and size of the lesion, and the bronchus reaching the lesion were ascertained Patients fasted for 6 hours before surgery. In surgery, patients were placed in a horizontal position, local anesthesia was administered to the nasal cavity and pharynx, and airway surface anesthesia was continued during the surgery to monitor patients' vital signs, such as heart rate, blood pressure, and oxygen saturation.

Patients received RP-EBUS treatment. According to the computerized tomography (CT) image results, the ultrasound probe was extended to the target bronchial lumen with the help of the bronchoscopic biopsy channel, and the surgeon opened the probe for scanning when he/she felt resistance. The scanning direction was perpendicular to the long axis of a patient's bronchus and was slowly rotated to achieve $360^{\circ}$ real-time images, during which, the image changes were observed until a lesion signal appeared; the 


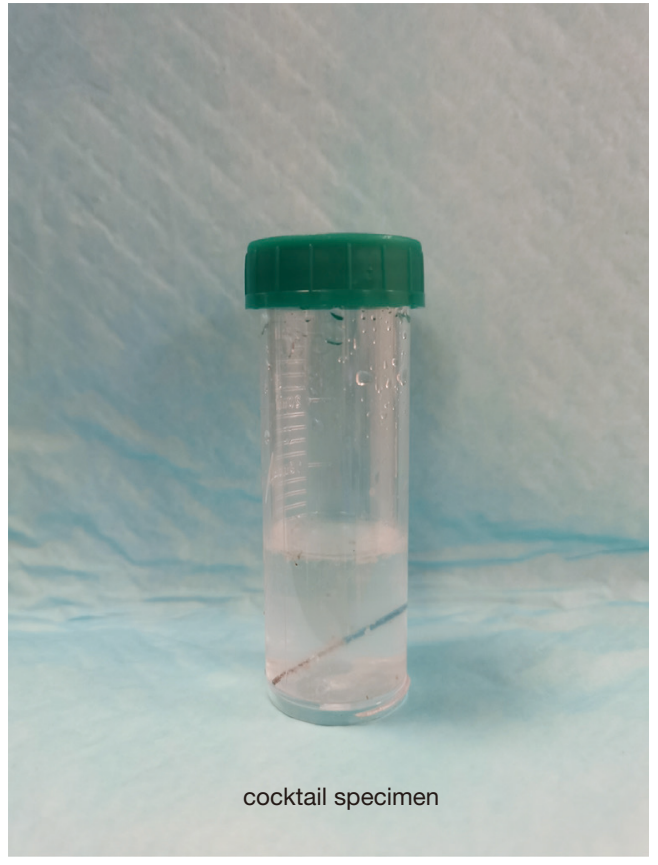

Figure 1 The "cocktail" sampling.

EBUS was then repeated in the adjacent bronchus to obtain clear images. After identifying the lesion site, the ultrasound probe was removed, and $\mathrm{TBBr}$ and BALF operations were performed along the path (the distance of which had been determined by the probe). The brush head taken by $\mathrm{TBBr}$ represents one or more sampling lines in the lung, while the BALF represents one or more sampling sectors or clusters in the lung.

In the $\mathrm{TBBr}$ group, only individual brush heads were collected from patients. The brush heads were cut into a sterile collection tube pre-filled with $1 \mathrm{~mL}$ of sterile saline with sterile scissors. In the BALF group, only lavage solution was collected from patients. No less than $5 \mathrm{~mL}$ of lavage solution was collected in a sterile collection tube. In the "cocktail" group, brush heads were first cut into a sterile collection tube with sterile scissors, $5 \mathrm{~mL}$ of lavage solution was then collected and poured into the same sterile collection tube (see Figure 1). All of the samples were stored at a low temperature $\left(4^{\circ} \mathrm{C}\right)$ for later use.

\section{Sequencing methods}

A respiratory tract sample $(600 \mu \mathrm{L})$ was taken from each patient (the viscous samples were liquefied by adding enzyme liquefaction solution). Each sample was processed using the pre-set procedure of the wall breaker. A DNA sample (of $300 \mu \mathrm{L}$ ) was extracted using TIANamp Micro DNA Kit (DP316, TIANGEN BIOTECH, Beijing, China) according to the kit instructions. The extracted DNA was transposonized to add adapter and establish libraries, which were prepared and then purified, amplified, and repurified. Qsep1 and Qubit were used to quantify the library fragment size and library concentration, respectively (Micro Far Cry Library Building Kit). The libraries were then mixed according to the preset onboard data volume and sequenced using the Nextseq 550Dx from Illumina, USA. At the same time, the pathogens were identified by combining clinical characteristics and excluding suspected human respiratory microflora. The scope of detection mainly included the database of the following known pathogens: 9,945 bacteria (including 144 mycobacteria and 107 mycoplasma or chlamydia), 6,760 viruses, 1,551 fungi, and 305 parasites. The mNGS test results of the specimens were analyzed comprehensively to identify the infectious pathogenic microorganisms. In the "cocktail" group, microbial DNA was extracted from $\mathrm{TBBr}$ and BALF, and then sequenced jointly on the machine in accordance with the "cocktail" mixing method. Specifically, the following mixing scheme was used: the $\mathrm{TBBr}$ brush head and the BALF collected from the target site was mixed and poured into a sterile collection tube, and the BALF group sent the bronchoalveolar lavage fluid collected from the target site were sent for mNGS analysis; the sterile brush heads taken from the target site in the TBBr group were sent for mNGS analysis.

\section{Criteria for a positive mNGS result}

When the coverage rate of bacteria (except Mycobacterium), virus or parasites was 10 times higher than that of any other microorganism, they were considered as positive pathogens (16). Mycobacteria were considered positive when at least 1 read or the mapping read number (genus or species level) was in the top 10 in the bacteria list $(17,18)$. mNGS identified fungi when its coverage rate scored 5 -fold higher than that of any other fun-gus (19).

\section{Data analysis}

Data were analyzed using SPSS 20.0 software, and counts were expressed as percentages (\%). Fisher's exact probability method was used to draw comparisons among the groups. The difference between the two tests was tested by 
Table 2 Comparison of the types of pathogens detected by mNGS in the 3 samples [n (\%)]

\begin{tabular}{lccc}
\hline Pathogen type $(\mathrm{n})$ & "Cocktail” group & TBBr group & BALF group \\
\hline 0 & $3(10.0)^{\star}$ & $11(36.7)^{\#}$ & $15(50.0)$ \\
1 & $13(43.3)^{\star}$ & $9(30.0)^{\#}$ & $5(16.7)$ \\
Multiple pathogens $(\geq 2)$ & $14(46.7)^{\star}$ & $10(33.3)$ & $10(33.3)$ \\
Positive samples & $27(90.0)^{\#}$ & $19(63.3)^{\#}$ & $15(50.0)$ \\
\hline
\end{tabular}

*, there was a significant difference between the "cocktail" group and the TBBr group $(\mathrm{P}<0.05)$; ", there was significant difference between the TBBr and BALF groups $(P<0.05)$. mNGS, metagenomic next-generation sequencing; TBBr, transbronchial brushing; BALF, bronchoalveolar lavage fluid.

McNemar's test. A $\mathrm{P}<0.05$ indicated a statistically significant difference.

\section{Results}

\section{Comparison of patbogen detection}

The mNGS analysis showed that the number of samples positive for pathogens in the "cocktail", TBBr, and BALF groups was $27(90.0 \%), 19(63.3 \%)$, and $15(50.0 \%)$ respectively. The sensitivity analysis showed that the "cocktail" group had a significantly higher than the $\mathrm{TBBr}$ group $(\mathrm{P}<0.05)$, and the $\mathrm{TBBr}$ group had a significantly higher than the BALF group $(\mathrm{P}<0.05)$. The detection of single pathogens was significantly higher in the "cocktail" group than in the $\mathrm{TBBr}$ group $(\mathrm{P}<0.05)$; and the detection of single pathogens was significantly higher in $\mathrm{TBBr}$ group than the BALF group $(\mathrm{P}<0.05)$. Multiple pathogen detection $(\geq 2)$ revealed that the "cocktail" group had a significantly higher number of multiple pathogens than the $\mathrm{TBBr}$ and the BALF groups $(\mathrm{P}<0.05)$; however, no such statistically significant difference was found between the TBBr and the BALF groups $(\mathrm{P}<0.05)$ (see Table 2).

\section{Pathogen detection situation analysis}

Figure 2 shows the chest computerized tomography (CT) scans, EBUS images, and the results of the mNGS detection for four patients in the "cocktail" group. With a $90 \%$ positive rate (and 27 cases), a wide variety of pathogens were detected by mNGS in the patients in the "cocktail" group. Further, a significantly higher number of multiple pathogens were detected in the "cocktail" group than in the TBBr and BALF groups (for details, see Table 3). Thus, the "cocktail" mix approach appears to have a number of advantages in the diagnosis of mixed infections.

\section{Effect of antibiotic exposure on pathogen detection}

The effect of antibiotic use on pathogen detection rate before mNGS testing was analyzed. The results showed that the positive rate of the groups using antibiotics and those without antibiotics had no significant effect $(\mathrm{P}>0.05)$, indicating that antibiotics had no significant effect on the detection rates of different sampling methods. However, given prior antibiotic patients, the positive rate of mNGS analysis was significantly higher in the "cocktail" group than in the TBBr group, $\mathrm{P}<0.05$; the $\mathrm{TBBr}$ group was significantly higher than the BALF group, $\mathrm{P}<0.05$ (see Table 4).

\section{Discussion}

Pulmonary infections have a high prevalence worldwide. Focal infection sites in the lung cannot be clearly observed; however, RP-EBUS is now used to localize local lesions, and thus provides great advantages for biopsies (20). The early identification of pathogens in patients provides an important reference that can be used to guide clinical medication, reduce high-risk factors, and develop accurate and effective treatment plans. Traditional etiological detection methods include biochemical, immunological, and sample culture methods; however, these methods are less sensitive, and the testing process is time-consuming and laborious (21). In addition, the pathogen detection rate is also affected by antibiotic treatments, which further increases the difficulty of detection. The mNGS technology can obtain accurate pathogen information based on a small number of pathological samples, which greatly improves the accuracy and efficiency of pathogen identification. In addition, the current mNGS samples are mostly $\mathrm{TBBr}$ single brush head or BALF lavage solution, which have a higher detection rate than traditional detection methods. 

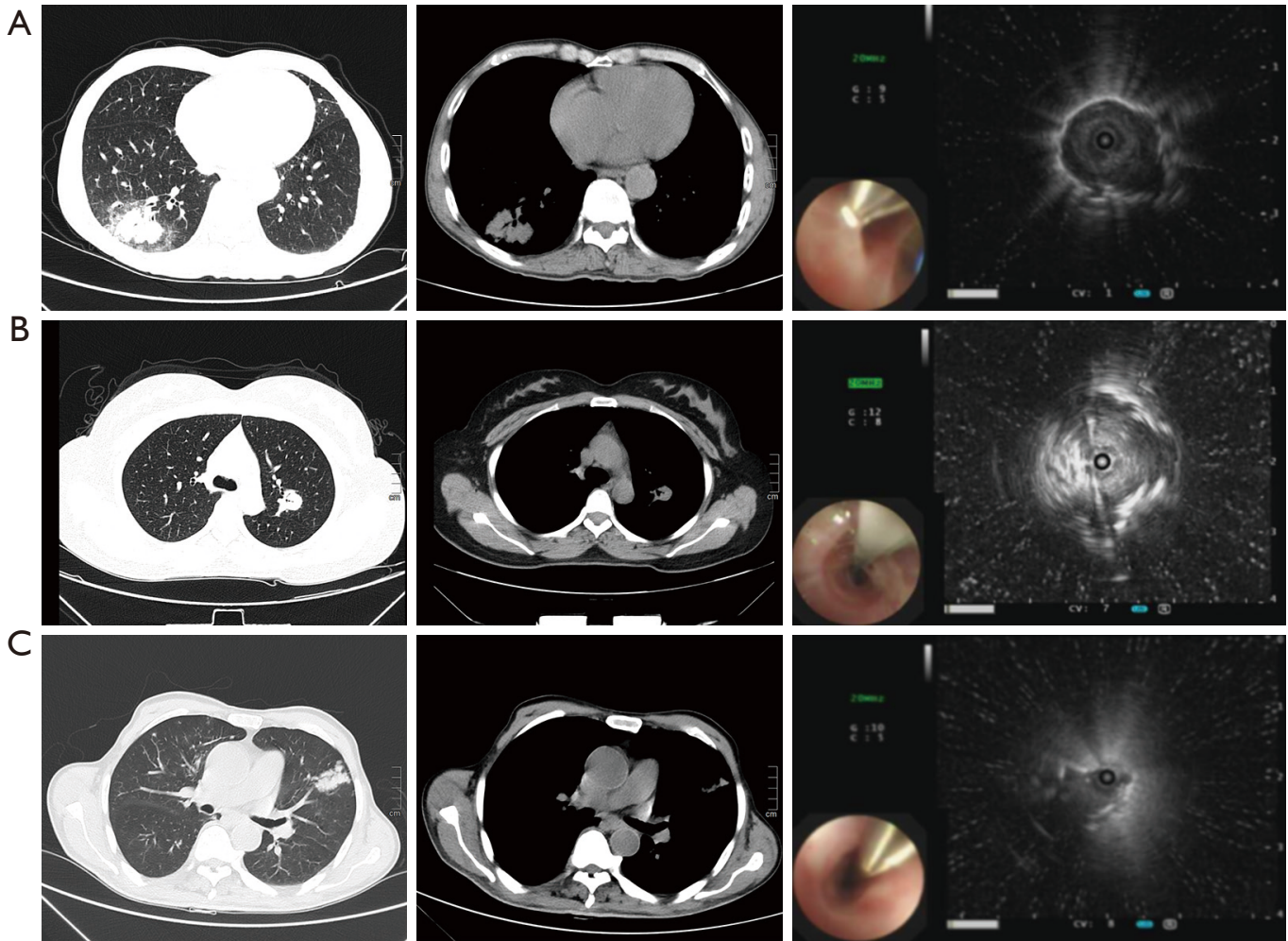

D
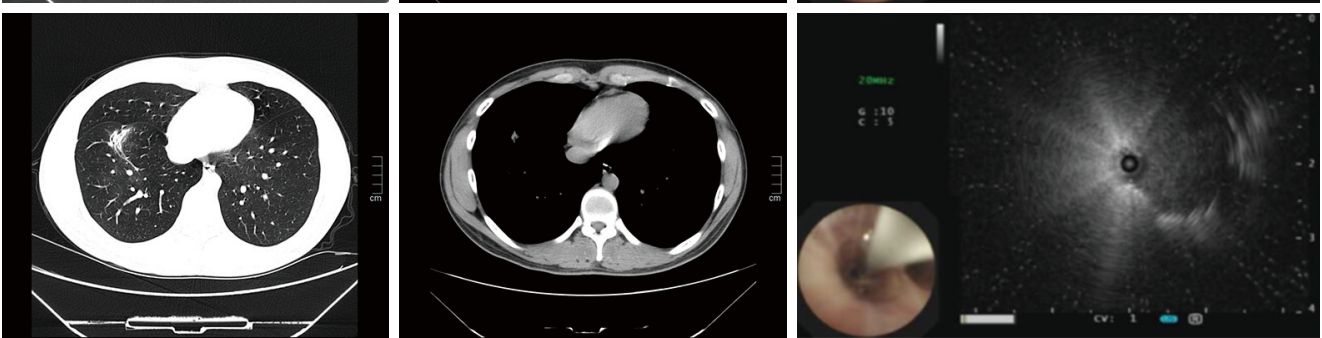

Figure 2 The chest computerized tomography (CT) scans, EBUS images and the results of mNGS detection of 4 patients in the "cocktail" specimen. (A) The mycobacterium tuberculosis complex was detected on the posterior apical foci of the left lung by mWS with detectable sequence number 169. (B) Human herpes virus type 6A, human herpes virus type 6A, human herpes virus type 5 (CMV) were detected by mNGS in the posterior basal segment of the right lower lobe of the lung with detection sequences of 275,8 and 4 , respectively. (C) Mycobacterium abscessus (CMV) was detected by mNGS in anterior basal segment lesions of right lower lung with detection sequence number 39158. (D) Mycobacterium tuberculosis complex was detected by mNGS in the anterior basal segment of the left upper lung lesion with detected sequence number 818. EBUS, endobronchial ultrasonography; mNGS, metagenomic next-generation sequencing.

It should be noted that the specimen range obtained by the transbronchial brushing ( $\mathrm{TBBr}$ ) group is smaller, the number of shedding cells obtained from bronchoalveolar lavage fluid (BALF) group are less, however the cost of multiple target detection samples will increase (22). To compensate for the above-mentioned shortcomings, this study chose a "cocktail" sample mixture for the mNGS analysis, which provides a reference for the application of mNGS in focal lung infections.
In this study, we systematically compared the diagnostic performance of mNGS with the "cocktail" mixture, individual brush-head sequencing, and lavage solution sequencing for pathogens. The results revealed that the sensitivity and positivity rate of multiple pathogen detection were as follows: "cocktail" mixture followed by TBBr brush followed by BALF lavage solution (i.e., the results showed that "cocktail" specimens have more advantages than other specimens in pathogen diagnosis). The "cocktail" mixture 
Table 3 Types of pathogens detected by mNGS in the 3 groups of patients

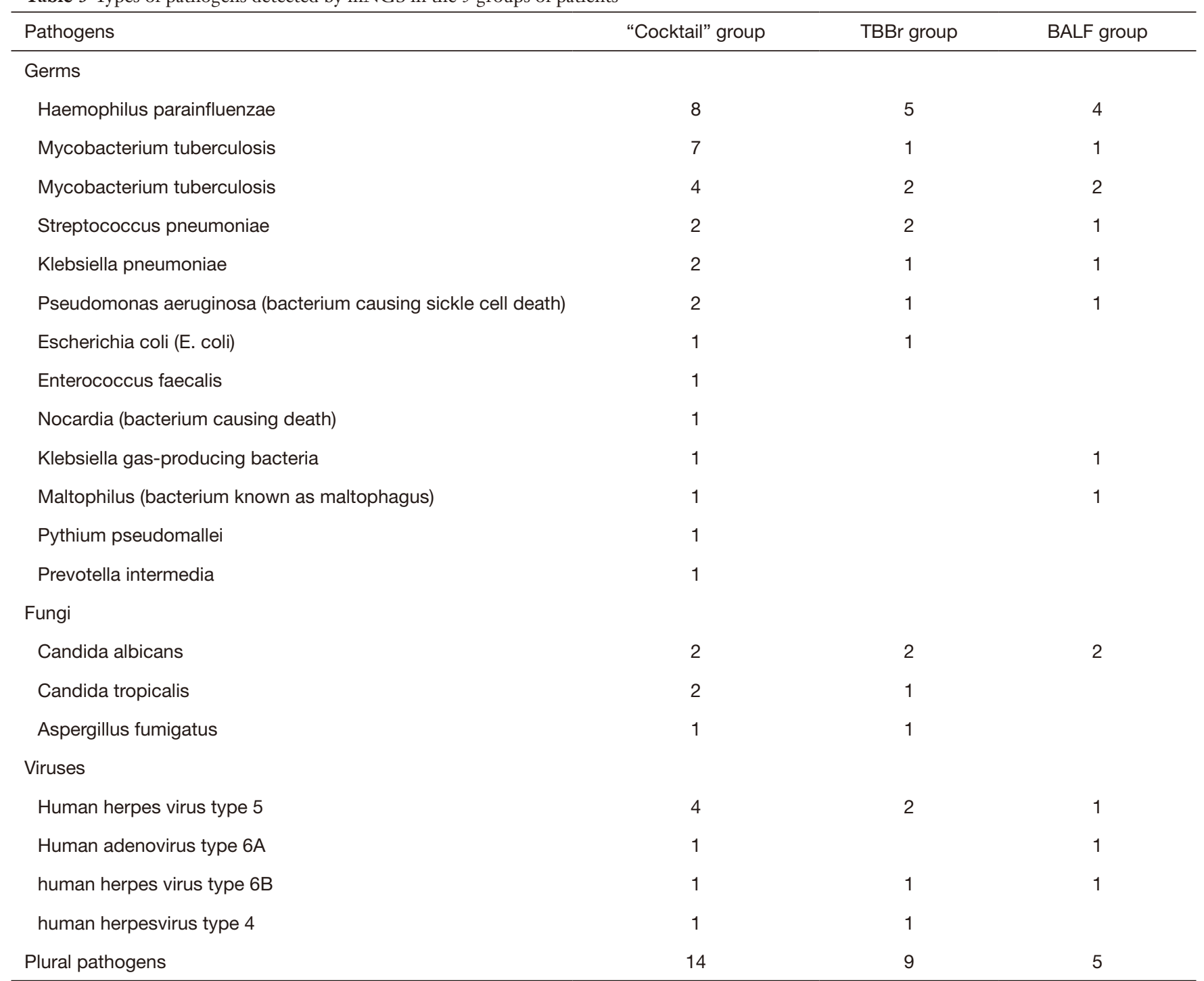

mNGS, metagenomic next-generation sequencing; TBBr, transbronchial brushing; BALF, bronchoalveolar lavage fluid.

Table 4 The effect of antibiotics on the detection rate of the mNGS analysis across the 3 samples

\begin{tabular}{|c|c|c|c|c|c|c|}
\hline Group (n) & \multicolumn{2}{|c|}{ “Cocktail” group } & \multicolumn{2}{|c|}{ TBBr group } & \multicolumn{2}{|c|}{ BALF group } \\
\hline Prior antibiotics [77] & 24 & 2 & 18 & 7 & 13 & 13 \\
\hline No prior antibiotics [13] & 4 & 1 & 3 & 1 & 2 & 2 \\
\hline
\end{tabular}

The positive rate of the groups using antibiotics and those without antibiotics had no significant effect ( $P>0.05)$, antibiotics had no significant effect on the detection rates of different sampling methods. mNGS, metagenomic next-generation sequencing. 
(followed by mNGS) had a high sensitivity to pathogen detection. These results have significant implications for the clinical formulation of targeted therapy. Additionally, Compared to patients who didn't receive antibiotics, antibiotic treatment has less influence on sequencing results, which can provide a reference for pathogen diagnoses in patients treated with antibiotics in clinical practice. The discrepancy in test results may be due to the smaller scope of pathology obtained by individual brush heads, and the fact that individual lavage fluid contains fewer exfoliated cells, which results in a lower mNGS positive detection rate. Similar to other reports (23), the pathogen identification analysis revealed that the predominant causative organism in the patients with focal lung infection was Gramnegative bacteria. It should be noted that the prevalence of mycobacterium tuberculosis infection in patients with pulmonary infection was approximately $40 \%$ (24), which was significantly higher than the results of this test. Thus, it appears that mNGS may be defective in the diagnosis of mycobacterium tuberculosis infection. Indeed, it may that insufficient mycobacterium tuberculosis wall breaking affects DNA extraction and results in a high false-negative bias (25). Metagenome second-generation sequencing (mNGS) using radial bronchoscopy cocktail specimens has a high pathogen detection rate, helps to reduce detection costs, is time saving, and can provide accurate information for clinical diagnosis, and the use of antibiotics had little effect on the results of the mNGS analysis. These clinically valuable results can be used in the diagnosis and treatment of patients with focal pulmonary infections.

This study had a number of shortcomings. First, detection efficiency may be affected by microbial adhesion to brush heads or tube walls during sample transportation. Second, as this was a retrospective study with a small sample size, there may be biases in the results. To further verify our conclusion, a well-designed prospective study with a larger sample size should be conducted.

\section{Conclusions}

Overall, the radial probe endobronchial ultrasound (RPEBUS) is able to clearly demonstrate local lesions on the pathway, and accurately obtain lesion specimens. mNGS has a number of advantages, including that it has a high pathogen detection rate, is time saving, and can provide accurate information for clinical diagnosis. The clinical value of the mNGS analysis of "cocktail" samples be taken under the non-real-time guidance of ultrasound instrumentation in the identification of pathogens, especially multiple pathogens, was higher than that of brush and lavage tests alone. In addition, the "cocktail" of mNGS has the unique advantage of providing a reference for the clinical application of mNGS in cases of pathogenic infections that are difficult to detect clinically, or in cases in which broad-spectrum antibiotics are widely used.

\section{Acknowledgments}

Funding: None.

\section{Footnote}

Reporting Checklist: The authors have completed the CONSORT reporting checklist. Available at http://dx.doi. org/10.21037/apm-20-2578

Data Sharing Statement: Available at http://dx.doi. org/10.21037/apm-20-2578

Conflicts of Interest: All authors have completed the ICMJE uniform disclosure form (available at http://dx.doi. org/10.21037/apm-20-2578). The authors have no conflicts of interest to declare.

Etbical Statement: The authors are accountable for all aspects of the work in ensuring that questions related to the accuracy or integrity of any part of the work are appropriately investigated and resolved. The study was conducted in accordance with the Declaration of Helsinki (as revised in 2013). The study was approved by Ethical Committee of Suzhou Municipal Hospital (No.: KL901157). All the patients and their families were informed of and agreed to participate in the study and signed the informed consent form.

Open Access Statement: This is an Open Access article distributed in accordance with the Creative Commons Attribution-NonCommercial-NoDerivs 4.0 International License (CC BY-NC-ND 4.0), which permits the noncommercial replication and distribution of the article with the strict proviso that no changes or edits are made and the original work is properly cited (including links to both the formal publication through the relevant DOI and the license). See: https://creativecommons.org/licenses/by-nc-nd/4.0/. 


\section{References}

1. Muthu V, Sehgal IS, Dhooria S, et al. Reversed Halo Sign on Radial EBUS Imaging. J Bronchology Interv Pulmonol 2018;25:e46-8.

2. Ni YN, Chen G, Sun J, et al. The effect of corticosteroids on mortality of patients with influenza pneumonia: a systematic review and meta-analysis. Crit Care 2019;23:99.

3. Rajapaksha P, Elbourne A, Gangadoo S, et al. A review of methods for the detection of pathogenic microorganisms. Analyst 2019;144:396-411.

4. Han D, Li Z, Li R, et al. mNGS in clinical microbiology laboratories: on the road to maturity. Crit Rev Microbiol 2019;45:668-85.

5. Chiu CY, Miller SA. Clinical metagenomics. Nature Public Health Emergency Collection 2019;20(6).

6. Magill SS, Edwards JR, Bamberg W, et al. Multistate point-prevalence survey of health care-associated infections. N Engl J Med 2014;370:1198-208.

7. Renaud C, Campbell AP. Changing epidemiology of respiratory viral infections in hematopoietic cell transplant recipients and solid organ transplant recipients. Curr Opin Infect Dis 2011;24:333-43.

8. Li H, Gao H, Meng H, et al. Detection of pulmonary infectious pathogens from lung biopsy tissues by metagenomic next-generation sequencing. Front Cell Infect Microbiol 2018;8:205.

9. Roumpeka DD, Wallace RJ, Escalettes F, et al. A Review of Bioinformatics Tools for Bio-Prospecting from Metagenomic Sequence Data. Front Genet 2017;8:23.

10. Wu B, Feng J, Zhang J. Principles for the application of diagnostic interventional pulmonology combined with pathogenic microbial metagenomics sequencing in pulmonary infection. Tianjin Med 2019;47:368-70.

11. Huang J, Jiang E, Yang D, et al. Metagenomic NextGeneration Sequencing versus Traditional Pathogen Detection in the Diagnosis of Peripheral Pulmonary Infectious Lesions. Infect Drug Resist 2020;13:567-76.

12. O'Brien JD, Ettinger NA, Shevlin D, et al. Safety and yield of transbronchial biopsy in mechanically ventilated patients. Crit Care Med 1997;25:440-6.

13. Leslie KO, Gruden JF, Parish JM, et al. Transbronchial biopsy interpretation in the patient with diffuse parenchymal lung disease. Arch Pathol Lab Med 2007;131:407-23.

14. Feng J, Wu B, Jiang E, et al. Pneumocystis jirovecii Pneumonia. In: Rapid On-Site Evaluation (ROSE) in Diagnostic Interventional Pulmonology. Singapore:
Springer, 2020:133-61.

15. Gutmann JL. Focal infection revisited-the swinging of the pendulum. Br Dent J 2017;62:81-92.

16. Langelier C, Zinter MS, Kalantar K, et al. Metagenomic sequencing detects res-piratory pathogens in hematopoietic cellular transplant patients. Am J Respir Crit Care Med 2018;197:524-8.

17. Simner PJ, Miller S, Carroll KC. Understanding the promises and hurdles of metagenomic next-generation sequencing as a diagnostic tool for infectious diseases. Clin Infect Dis 2018; 66:778-88.

18. van Ingen J, Kohl TA, Kranzer K, et al. Global outbreak of severe Mycobacterium chimaera disease after cardiac surgery: a molecular epidemiological study. Lancet Infect Dis 2017;17:1033-41.

19. Schlaberg R, Chiu CY, Miller S, et al. Validation of metagenomic next-generation sequencing tests for universal pathogen detection. Arch Pathol Lab Med 2017;141:776-86.

20. Brown JR, Bharucha T, Breuer J. Encephalitis diagnosis using metagenomics: application of next generation sequencing for undiagnosed cases. J Infect 2018;76:225-40.

21. Jiang LX, Ren HY, Zhou HJ, et al. Progress in research of detection assay for pathogens causing community acquired pneumonia. Zhonghua Liu Xing Bing Xue Za Zhi 2016;37:1051-4.

22. Villarroel J, Larsen MV, Kilstrup M, et al. Metagenomic Analysis of Therapeutic PYO Phage Cocktails from 1997 to 2014. Viruses 2017;9:328.

23. Cillóniz C, Dominedò C, Torres A. Multidrug Resistant Gram-Negative Bacteria in Community-Acquired Pneumonia. Crit Care 2019;23:79.

24. Wei M, Zhao Y, Qian Z, et al. Pneumonia caused by Mycobacterium tuberculosis. Microbes Infect 2020;22:278-84.

25. Eddabra R, Ait Benhassou H. Rapid molecular assays for detection of tuberculosis. Pneumonia (Nathan) 2018;10:4.

(English Language Editor: L. Huleatt)

Cite this article as: Chen Y, Yan X, Li N, Zhang Q, Lv Y, Ruan T. Metagenomic next-generation sequencing of radial ultrasound bronchoscopy-guided "cocktail” specimens as an efficient method for the diagnosis of focal pulmonary infections: a randomised study. Ann Palliat Med 2021;10(2):2080-2088. doi: 10.21037/apm-20-2578 